\title{
Generalized Counting for Lifted Variable Elimination
}

\author{
Nima Taghipour, Jesse Davis, and Hendrik Blockeel \\ Department of Computer Science, KU Leuven, Belgium
}

\begin{abstract}
Lifted probabilistic inference methods exploit symmetries in the structure of probabilistic models to perform inference more efficiently. In lifted variable elimination, the symmetry among a group of interchangeable random variables is captured by counting formulas, and exploited by operations that handle such formulas. In this paper we generalize the structure of counting formulas and present a set of inference operators that introduce and eliminate these formulas from the model. This generalization expands the range of problems that can be solved in a lifted way. Our work is closely related to the recently introduced method of joint conversion. Due to its more fine grained formulation, however, our approach can provide more efficient solutions than joint conversion.
\end{abstract}

\section{Introduction}

Probabilistic logical languages combine elements of first-order logic with graphical models to succinctly model complex, uncertain, structured domains [3]. These domains often involve a large number of objects, making efficient inference a major challenge. To address this problem, Poole [5] introduced the concept of lifted probabilistic inference, i.e., inference that exploits the symmetries in the structure of the model to gain efficiency. Lifted inference methods use two main techniques or tools for lifting: (1) divide the problem into isomorphic subproblems, solve one instance, and aggregate the result, and (2) count the number of isomorphic configurations for a group of interchangeable objects, instead of enumerating all possible configurations. Our focus in this paper is on the second tool, counting, in the context of lifted variable elimination (LVE) $[5,2,4]$.

LVE uses counting formulas to capture the interchangeability among objects [4]. A counting formula aggregates the joint state of a group of random variables into histograms that show only the number of variables with each state. For instance, the formula $\#_{X}[\operatorname{Attends}(X)]$, captures the number of people who attend a workshop, without distinguishing between their identity. As the number of possible aggregate states (histograms) is much smaller than the number of joint states of the group, lifted operations achieve large efficiency gains by directly manipulating counting formulas, instead of the individual variables.

Counting formulas, as used so far in LVE [4], have specific syntactic restrictions. Some of these restrictions are not fundamental, and can be removed to arrive at more general counting formulas, which yields more opportunities for 
lifting. One such restriction is that a counting formula contains only a single atom, i.e., it aggregates the state of a group of individual random variables. In this paper, first we generalize the definition of counting formulas in a straightforward way, to allow a counting formula to aggregate the state of a group of tuples of random variables. For instance, we allow a counting formula such as $\#_{X}[\operatorname{Attends}(X), \operatorname{Presents}(X)]$ that counts the number of people that (do not) attend and (do not) present a paper at the workshop. Second, we present a set of inference operators that introduce and manipulate these generalized formulas, and show that these expand the opportunities for lifting, and hence for more efficient inference, compared to the original formulation of counting operations.

Our work is closely related to Apsel and Brafman's [1] on joint conversion and just-different counting conversion. However, our method uses a more fine grained formulation, and can offer more efficient solutions than joint conversion.

\section{Representation}

Like earlier work on LVE $[2,4,5]$, we use a representation based on parametrized random variables and parametric factors. This representation combines random variables and factors (as used in factor graphs) with concepts from logic. The goal is to compactly define complex probability distributions over many variables.

We use the term 'variable' in both the logical and probabilistic sense. We use logvar for logical variables and randvar for random variables. We write variables in uppercase and values in lowercase.

Preliminaries. A factor $f=\phi_{f}\left(\mathcal{A}_{f}\right)$, where $\mathcal{A}_{f}=\left(A_{1}, \ldots, A_{n}\right)$ are randvars and $\phi_{f}$ is a potential function, maps each configuration of $\mathcal{A}_{f}$ to a real. A factor graph is a set of factors $F$ over randvars $\mathcal{A}=\bigcup_{f \in F} \mathcal{A}_{f}$ and defines a probability distribution $\mathcal{P}_{F}(\mathcal{A})=\frac{1}{Z} \prod_{f \in F} \phi_{f}\left(\mathcal{A}_{f}\right)$, with $Z$ a normalization constant.

The vocabulary consists of predicates (representing properties and relations), constants (representing objects) and logvars. Each logvar $X$ has a finite domain $\mathcal{D}(X)$, which is a set of constants $\left\{x_{1}, \ldots, x_{n}\right\}$. A constraint $C$ on a set of logvars $\mathbf{X}=\left\{X_{1}, \ldots, X_{n}\right\}$ is a conjunction of inequalities of the form $X_{i} \neq t$ where $t$ is a constant in $\mathcal{D}\left(X_{i}\right)$ or a logvar in $\mathbf{X}$. A substitution $\theta=\left\{X_{1} \rightarrow t_{1}, \ldots, X_{n} \rightarrow\right.$ $\left.t_{n}\right\}$ maps logvars to terms. When all $t_{i}$ 's are constants, $\theta$ is called a grounding substitution. Given a constraint $C$, we use $\operatorname{gr}(\mathbf{X} \mid C)$ to denote the set of grounding substitutions to $\mathbf{X}$ that are consistent with $C$.

Parametrized randvars. The representation associates atoms with randvars. For this, every predicate is assigned a range, i.e., a set of possible values, e.g., range $($ BloodType $)=\{a, b, a b, o\}$. A ground atom then represents a randvar, e.g., BloodType(joe). To compactly encode distributions over many randvars, Parametrized randvars (PRV) were introduced [5]. A PRV is of the form $P(\mathbf{X}) \mid C$, where $P(\mathbf{X})$ is an atom and $C$ is a constraint on $\mathbf{X}$. A PRV $\mathcal{V}=P(\mathbf{X}) \mid C$ represents (or covers) the set of randvars $R V(\mathcal{V})=\{P(\mathbf{X}) \theta \mid \theta \in \operatorname{gr}(\mathbf{X} \mid C)\}$.

Example. The PRV $\mathcal{V}=\operatorname{Smokes}(X) \mid X \neq x_{1}$, with $D(X)=\left\{x_{1}, \ldots, x_{n}\right\}$, represents $n-1$ randvars $R V(\mathcal{V})=\left\{\operatorname{Smokes}\left(x_{2}\right), \ldots \operatorname{Smokes}\left(x_{n}\right)\right\}$. 
Parametric factors (parfactors). Like PRVs compactly encode sets of randvars, parfactors compactly encode sets of factors. A parfactor is of the form $\forall \mathbf{L}: \phi(\mathcal{A}) \mid C$, with $\mathbf{L}$ a set of logvars, $C$ a constraint on $\mathbf{L}, \mathcal{A}=\left(A_{i}\right)_{i=1}^{n}$ a sequence of atoms parametrized with $\mathbf{L}$, and $\phi$ a potential function on $\mathcal{A}$. The set of logvars occurring in $\mathcal{A}$ is denoted logvar $(\mathcal{A})$, and we have logvar $(\mathcal{A}) \subseteq \mathbf{L}$. When logvar $(\mathcal{A})=\mathbf{L}$, we omit $\mathbf{L}$ and write the parfactor as $\phi(\mathcal{A}) \mid C$. A factor $\phi\left(\mathcal{A}^{\prime}\right)$ is called a grounding of a parfactor $\phi(\mathcal{A}) \mid C$ if $\mathcal{A}^{\prime}$ can be obtained by instantiating $\mathbf{L}$ according to a grounding substitution $\theta \in \operatorname{gr}(\mathbf{L} \mid C)$. The set of all groundings of a parfactor $g$ is denoted $g r(g)$.

Example. Parfactor $g=\phi_{1}(\operatorname{Smokes}(X))$ represents the set of $n$ ground factors $\operatorname{gr}(g)=\left\{\phi_{1}\left(\operatorname{Smokes}\left(x_{1}\right)\right), \ldots, \phi_{1}\left(\operatorname{Smokes}\left(x_{n}\right)\right)\right\}$.

Parfactor models. When talking about a model below, we mean a set of parfactors. In essence, a set of parfactors $G$ is a compact way of defining a set of factors $F=\{f \mid f \in \operatorname{gr}(g) \wedge g \in G\}$. The corresponding probability distribution is $\mathcal{P}_{G}(\mathcal{A})=\frac{1}{Z} \prod_{f \in F} \phi_{f}\left(\mathcal{A}_{f}\right)$.

Following the literature, and to ease the exposition, we assume that the model is in normal form [4]. Note that any model can be rewritten into an equivalent one in normal form using the auxiliary operations [4].

\section{Lifted Variable Elimination}

The state of art in lifted variable elimination (LVE) is the result of various complementary efforts $[5,4,1,2]$. In this section we briefly review the C-FOVE [4] algorithm, which forms the basis of our work. Another extension to C-FOVE, namely joint formulas [1], is discussed and compared with in Section 7.

Variable elimination calculates a marginal distribution by eliminating randvars in a specific order from the model until reaching the desired marginal [6]. To eliminate a single randvar $V$, it first multiplies all the factors containing $V$ into a single factor and then sums out $V$ from that single factor. LVE does this on a lifted level by eliminating parametrized randvars (i.e., whole groups of randvars) from parfactors (i.e., group of factors). The outer loop of LVE is as follows.

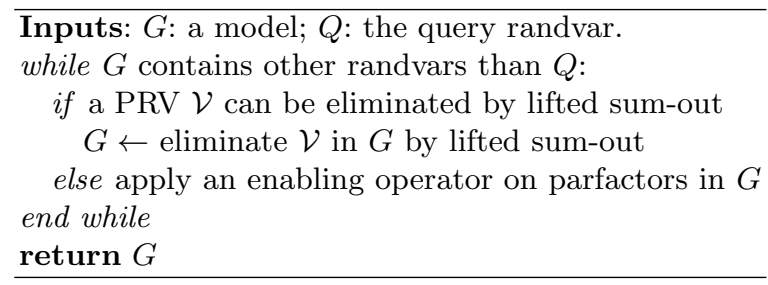

As this shows, LVE performs inference using a set of lifted operators:

Lifted sum-out sums-out a PRV, and hence all the randvars represented by that PRV, from the model. Lifted sum-out is applicable only under a precondition (each randvar represented by the PRV appears in exactly one grounding of exactly one parfactor in the model). The goal of all other operators is to manipulate the parfactors into a form that satisfies this precondition. In this sense, all operators except lifted sum-out are enabling operators. 
Lifted multiplication performs the equivalent of many factor multiplications in one lifted operation. It prepares the model for sum-out by replacing all the parfactors that share a particular PRV by a single equivalent product parfactor. Splitting and shattering rewrite the model into a normal form in which, e.g., each pair of PRVs represent either identical or disjoint randvars.

Counting conversion is another important enabling operator, which introduces counting formulas into the model. We introduce and generalize counting formulas, along with operations that handle them, in the following sections.

\section{Generalized Counting Formulas}

Counting formulas aggregate the state of a group of interchangeable randvars into histograms that show the number of randvars with each value. They thus lift the computations to the level of the aggregate state of the group, without considering the individuals. This speeds up inference as the number of possible aggregate states (histograms) is polynomial in the group size, whereas the number of joint states is exponential. In this section, we generalize counting formulas, such that they aggregate the state of a group of tuples of atoms, instead of individual atoms. This permits lifting in cases where not all individual randvars are interchangeable, but specific tuples of randvars are.

Counting formulas. We define a counting formula $(\mathrm{CF})$ to be of the form $\gamma=\#_{X: C}\left[P_{1}\left(\mathbf{X}_{1}\right), \ldots, P_{k}\left(\mathbf{X}_{k}\right)\right]$, with $C$ a constraint on the counted logvar $X$, and $X \in \mathbf{X}_{i}(i=1, \ldots, k)$. The logvar $X$ is bound by the formula and excluded from logvar $(\gamma)$. In a grounded $C F$ all terms except the counted logvar are constants. Such a formula represents a counting randvar $(C R V)$ whose range is the set of possible histograms that distribute $n$ elements into $r=$ $\prod_{i=1}^{k}\left|\operatorname{range}\left(P_{i}\right)\right|$ buckets. Each histogram $h=\left\{\left(r_{i}, n_{i}\right)\right\}_{i=1}^{r}$ shows for each $r_{i} \in$ $\times_{i=1}^{k}$ range $\left(P_{i}\right)$ the number $n_{i}$ of tuples $\left(P_{1}(\ldots, x, \ldots), \ldots, P_{k}(\ldots, x, \ldots)\right)$ whose state is $r_{i}$. The state of the CRV is thus determined by the state of the randvars $\cup_{i=1}^{k} R V\left(P_{i}(\ldots, X, \ldots) \mid C\right)$. For simplicity, we denote a histogram $h=\left\{\left(r_{i}, n_{i}\right)\right\}_{i=1}^{r}$ by the list of counts $\left(n_{1}, \ldots, n_{r}\right)$, when $r_{i}$ are apparent from the context.

Example. Having $D(X)=\{a n n$, bob, carl, dave $\}$, the counting randvar $\gamma=$ $\#_{X}[\operatorname{Smokes}(X), \operatorname{Asthma}(X)]$ covers tuples of randvars $R V(\gamma)=\left\{\left(\operatorname{Smokes}\left(x_{i}\right)\right.\right.$, Asthma $\left.\left.\left(x_{i}\right)\right) \mid x_{i} \in D(X)\right\}$. Assume $R V(\gamma)$ are assigned the following values:

\begin{tabular}{c|cc}
$X$ & Smokes $(X)$ & Asthma $(X)$ \\
\hline ann & $f$ & $t$ \\
bob & $f$ & $f$ \\
carl & $f$ & $f$ \\
dave & $t$ & $t$
\end{tabular}

Then the value of the CRV is the histogram $h=\{(t t, 1),(t f, 0),(f t, 1),(f f, 2)\}$.

Our definition of a $\mathrm{CF}$, which allows a tuple of atoms in the formula, is a simple generalization of Milch et al.'s definition [4], which only allows a single atom (a 1-tuple). We show how these more general CFs create more opportunities for lifting through a suite of model conversion operations, in Section 5, and present a lifted sum-out operation for them in Section 6 . 


\section{Conversion Operations}

In this section we present conversion operations that rewrite the model in terms of counting formulas. The first operation is a generalization of C-FOVE's counting conversion [4], while the rest are new operations. Throughout this paper, we illustrate the application of the new operators on examples for which C-FOVE [4] has no lifted solution. As such, we show how the new operations perform inference with complexity polynomial in the domain size, where C-FOVE cannot avoid the exponential complexity of propositional inference.

\subsection{Counting Conversion}

Counting formulas (CFs) are introduced into the model by counting conversion. By rewriting the model (replacing an atom) with a $\mathrm{CF}$, this operation allows us to compactly represent and manipulate a high dimensional factor on a set of interchangeable randvars. It achieves the equivalent of multiplying groundings of a single parfactor with each other, and thus functions as an enabling operation for lifted sum-out. We generalize this operation to a rewrite rule that replaces a tuple of atoms with a CF. By removing the restriction on the number of counted atoms, this generalization provides more opportunities for lifting.

Example. Consider the parfactor $\phi(A(X), B(X), C(Y), D(Y))$. Lifted sum-out is not applicable here, as no atom contains all the logvars. Counting conversion removes a logvar from the set of free logvars, hence preparing the model for lifted sum-out. Here we perform counting conversion on logvar $X$ by introducing a $\mathrm{CF}$ on the tuple of atoms $A(X), B(X)$, and rewrite the parfactor as $\phi^{\prime}\left(\#_{X}[A(X), B(X)], C(Y), D(Y)\right)$, where $\phi^{\prime}$ is such that for each histogram $h($. in the range of $\#_{X}[A(X), B(X)], \phi^{\prime}(h(), c, d$.$) is equal to$

$$
\phi(t, t, c, d)^{h(t t)} \cdot \phi(t, f, c, d)^{h(t f)} \cdot \phi(f, t, c, d)^{h(f t)} \cdot \phi(f, f, c, d)^{h(f f)}
$$

Note that after this conversion, logvar $Y$ is the only free logvar in the parfactor, which enables lifted sum-out of $C(Y)$ and $D(Y) .{ }^{1}$

Operation: COUNT-CONVERT

Input: (1) a parfactor $g=\forall \mathbf{L}: \phi(\mathcal{A}) \mid C(2)$ a logvar $X \in \operatorname{logvar}(\mathcal{A})$

Preconditions: (1) there is no counting formula in the set $\mathcal{A}_{X}=\{A \in \mathcal{A} \mid X \in$ logvar $(A)\}$ (2) there is no counting formula $\gamma=\#_{X_{i}: C_{i}}[\ldots]$ in $\mathcal{A}$, such that $\left(X_{i} \neq X\right) \in C_{i}$

Output: $g^{\prime}=\forall \mathbf{L}^{\prime}: \phi^{\prime}\left(\mathcal{A}^{\prime}\right) \mid C^{\prime}$, such that:

(1) $\mathbf{L}^{\prime}=\mathbf{L} \backslash\{X\}(2) C^{\prime}$ is the projection of $C$ on $\mathbf{L}^{\prime}(3) \mathcal{A}^{\prime}=\mathcal{A} \backslash \mathcal{A}_{X} \cup\left\{\#_{X}\left[\mathcal{A}_{X}\right]\right\}$, and (4) for each valuation $(h(),. \mathbf{a})$ to $\left(\#_{X}\left[\mathcal{A}_{X}\right], \mathcal{A} \backslash \mathcal{A}_{X}\right)$ :

$$
\phi^{\prime}(h(.), \mathbf{a})=\prod_{\mathbf{a}^{\prime} \in \operatorname{range}\left(\mathcal{A}_{X}\right)} \phi\left(\mathbf{a}^{\prime} ; \mathbf{a}\right)^{h\left(\mathbf{a}^{\prime}\right)}
$$

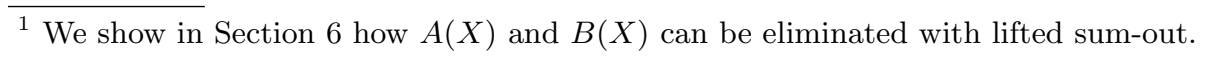


The preconditions for counting conversion of a logvar $X$ require that it does not appear inside an existing CF. This means that $X$ cannot appear (1) in an atom in a $\mathrm{CF}$, or (2) in the constraint associated with a CF. Excluding the first case ensures that the result of conversion can be represented by our CFs, and does not require more complicated structures like nested or overlapping CFs, which are not well defined in our formulation. In the second case, where $X$ is in a constraint with a counted logvar, counting conversion is still possible, but by the operation of merge-counting, which we introduce in Section 5.3. These preconditions for counting conversion are weaker than that of C-FOVE, which requires $X$ to appear in exactly one atom in the parfactor [4].

\subsection{Merging Counting Formulas}

Two CFs can count over tuples of randvars with overlapping randvars between them. When such formulas appear in a parfactor together, to sum-out the common randvars, we need to first merge the formulas into one.

Example. Consider the parfactor $\phi\left(\#_{X}[S(X)], \#_{Y}[S(Y), A(Y)]\right)$. The first argument, $\gamma_{1}=\#_{X}[S(X)]$, aggregates the state of randvars $R V(S(X))$, and the second argument, $\gamma_{2}=\#_{Y}[S(Y), A(Y)]$, the state of $R V(S(X), A(X))$. As the second group of randvars is a superset of the first set, given a histogram $h_{2}($. for $\gamma_{2}$, we can infer the value $h_{1}$ of $\gamma_{1}$. We can therefore merge the two CFs into one $\#_{X}[S(X), A(X)]$ and rewrite the parfactor as $\phi^{\prime}\left(\#_{X}[S(X), A(X)]\right)$ where $\phi^{\prime}\left(h_{2}\right)=\phi\left(h_{1}, h_{2}\right)$ and $h_{1}$ is the histogram that results from projecting $h_{2}$ on the assignments to the $S$ randvars. Concretely, having the counts $\left(n_{t t}, n_{t f}, n_{f t}, n_{f f}\right)$ for $h_{2}$, the value of $h_{1}$ is the histogram with counts $\left(n_{t t}+n_{t f}, n_{f t}+n_{f f}\right)$.

Although in the above example one CRV was a superset of the other, merging can be applied to any pair of formulas with overlapping randvars. For instance merging rewrites the parfactor $\left.\phi\left(\#_{X}[A(X), B(X)], \#_{Y}[B(Y), C(Y))\right]\right)$ into $\phi\left(\#_{X}[A(X), B(X), C(X)]\right)$. To formalize this operator we introduce the notion of compatible valuations to atoms, and the projection of histograms.

A pair of valuations $\left(\mathbf{a}_{1}, \mathbf{a}_{2}\right)$ to $\left(\mathcal{A}_{1}, \mathcal{A}_{2}\right)$ are compatible, denoted by $\mathbf{a}_{1} \sim \mathbf{a}_{2}$, if each atom $A_{i} \in \mathcal{A}_{1} \cap \mathcal{A}_{2}$ is assigned with the same value $a_{i}$ in both $\mathbf{a}_{1}$ and $\mathbf{a}_{2}$.

Given a counting formula $\gamma=\#_{X}[\mathcal{A}]$, the projection of a histogram $h \in$ range $(\gamma)$ on $\mathcal{A}^{\prime} \subseteq \mathcal{A}$, is a histogram $h^{\prime} \in \operatorname{range}\left(\#_{X}\left[\mathcal{A}^{\prime}\right]\right)$ s.t. for each $\mathbf{a}^{\prime} \in$ range $\left(\mathcal{A}^{\prime}\right): h^{\prime}\left(\mathbf{a}^{\prime}\right)=\sum_{\mathbf{a} \sim \mathbf{a}^{\prime}} h(\mathbf{a})$. We denote the projection of $h$ on $\mathcal{A}^{\prime}$ by $h_{\left[\mathcal{A}^{\prime}\right]}$.

\section{Operation: MERGE}

Input: (1) a parfactor $g=\forall \mathbf{L}: \phi(\mathcal{A}) \mid C(2)$ a pair of counting formulas $\left(\gamma_{1}, \gamma_{2}\right)=$ $\left(\#_{X_{1}: C_{1}}\left[\mathcal{A}_{1}\right], \#_{X_{2}: C_{2}}\left[\mathcal{A}_{2}\right]\right)$ in $\mathcal{A}$

Precondition: $\operatorname{gr}\left(X_{1}: C \wedge C_{1}\right)=\operatorname{gr}\left(X_{2}: C \wedge C_{1}\right)$

Output: $g^{\prime}=\forall \mathbf{L} \cdot \phi^{\prime}\left(\mathcal{A}^{\prime}\right) \mid C$, such that:

(1) $\mathcal{A}^{\prime}=\mathcal{A} \backslash\left\{\gamma_{1}, \gamma_{2}\right\} \cup\left\{\#_{X_{1}}\left[\mathcal{A}_{12}\right]\right\}$, with $\mathcal{A}_{12}=\mathcal{A}_{1} \cup \mathcal{A}_{2} \theta$ and $\theta=\left\{X_{2} \rightarrow X_{1}\right\}$

(2) for each valuation $(h(),. \mathbf{a})$ to $\left(\#_{X_{1}}\left[\mathcal{A}_{12}\right], \mathcal{A} \backslash\left\{\gamma_{1}, \gamma_{2}\right\}\right)$ :

$$
\phi^{\prime}(h, \mathbf{a})=\phi\left(h_{\left[\mathcal{A}_{1}\right]}, h_{\left[\mathcal{A}_{2} \theta\right]} ; \mathbf{a}\right)
$$




\subsection{Merge-counting}

This operation is applicable when counting conversion cannot replace a tuple of atoms by a new CF, but needs to merge them into an existing CF. This happens during counting conversion on a logvar $X$ that is in the same parfactor with a $\mathrm{CF} \#_{Y: Y \neq X}\left[\mathcal{A}_{Y}\right]$, whose counted logvar is in an inequality constraint with $X$. Here, we cannot rewrite the parfactor using two separate formulas $\#_{Y}\left[\mathcal{A}_{Y}\right]$ and $\#_{X}\left[\mathcal{A}_{X}\right]$. This is because the histograms of these two CFs do not determine the value of the original parfactor. Instead, we can apply merge-counting, which incorporates the atoms $\mathcal{A}_{X}$ inside the existing formula. The following example demonstrates this operation.

Example. Consider the parfactor $g$ of the form $\phi\left(\#_{X: X \neq Y}[A(X)], B(Y)\right)$. We show how merge-counting on logvar $Y$ rewrites $g$ as an equivalent parfactor $g^{\prime}=\phi^{\prime}\left(\#_{X}[A(X), B(X)]\right)$. For this we need to properly define the potential $\phi^{\prime}$ based on $\phi$. Note that $g^{\prime}$ represents a single factor, while $g$ represents $n$ factors, one for each $y \in D(Y)$. The potential $\phi^{\prime}$ should thus be defined such that $g^{\prime}$ evaluates the product of these $n$ factors, for any valuation of randvars $A(X)$ and $B(X)$. Consider a valuation that yields histogram $h($.$) for \#_{X}[A(X), B(X)]$. To compute $\phi^{\prime}(h)$, we compute the value of $g$ at this valuation, based on the following observations: in $g r(g)$, each factor $g_{y}=\phi\left(\gamma_{y}, B(y)\right)$, has a distinct counting formula $\gamma_{y}=\#_{X: X \neq y}[A(X)]$, which covers all the randvars $R V(A(X))$ except $A(y)$, due to the inequality constraint. Since the histogram of $\#_{X}[A(X)]$ is $h_{[A]}=\left(n_{t}, n_{f}\right)$, each CRV $\gamma_{y}$, which excludes the value of one randvar $A(y)$, takes on one of the two histograms

$$
\begin{aligned}
& -h_{[A]}^{-t}=\left(n_{t}-1, n_{f}\right), \text { when } A(y)=t, \text { or } \\
& -h_{[A]}^{-f}=\left(n_{t}, n_{f}-1\right), \text { when } A(y)=f
\end{aligned}
$$

Each factor $g_{y}$ in $\operatorname{gr}(g)$ thus evaluates to one of the four values $\phi\left(h^{-}, b\right)$, for $\left(h^{-}, b\right) \in\left\{h_{[A]}^{-t}, h_{[A]}^{-f}\right\} \times\{t, f\}$. Knowing the number $\#\left(h^{-}, b\right)$ of factors with each value $\phi\left(h^{-}, b\right)$, we can compute the desired potential $\phi^{\prime}$ as:

$$
\phi^{\prime}(h(.))=\prod_{\left(h^{-}, b\right)} \phi\left(h^{-}, b\right)^{\#\left(h^{-}, b\right)}
$$

The numbers $\#\left(h^{-}, b\right)$ are inferred directly from $h($.$) . For instance, \#\left(h_{[A]}^{-t}, t\right)$, the number of $y$ s with $\gamma_{y}=h_{[A]}^{-t}$ and $B(y)=t$, by definition equals $h(t t)$, that is, the number of $y$ s with $A(y)=B(y)=t$. With similar reasoning, we determine all the numbers $\#\left(h^{-}, b\right)$ from $h($.$) and define the desired potential \phi^{\prime}$ as:

$$
\phi^{\prime}(h(.))=\phi\left(h_{[A]}^{-t}, t\right)^{h(t t)} \cdot \phi\left(h_{[A]}^{-f}, t\right)^{h(f t)} \cdot \phi\left(h_{[A]}^{-t}, f\right)^{h(t f)} \cdot \phi\left(h_{[A]}^{-f}, f\right)^{h(f f)}
$$

As such, merge-counting replaces the parfactor $g$ with the equivalent parfactor $g^{\prime}=\phi^{\prime}\left(\#_{X}[A(X), B(X)]\right)$, by directly computing $\phi^{\prime}$ from $\phi$. 
Operation: MERGE-COUNT

Input: (1) a parfactor $g=\forall \mathbf{L}: \phi(\mathcal{A}) \mid C$ (2) a counting formula $\gamma=\#_{X_{1}: C_{1}}\left[\mathcal{A}_{1}\right]$ in $\mathcal{A}(3)$ a logvar $X_{2}$ in $\log v a r(\mathcal{A})$

Precondition: (1) there is no counting formula in $\mathcal{A}_{2}=\left\{A \in \mathcal{A} \mid X_{2} \in \operatorname{logvar}(A)\right\}$

(2) $\gamma$ is the only counting formula whose counted logvar $X_{1}$ is in an inequality constraint with $X_{2}$

Output: $g^{\prime}=\forall \mathbf{L}^{\prime}: \phi^{\prime}\left(\mathcal{A}^{\prime}\right) \mid C^{\prime}$, such that:

(1) $\mathbf{L}^{\prime}=\mathbf{L} \backslash\left\{X_{2}\right\}$ (2) $C^{\prime}$ is the projection of $C$ on $\mathbf{L}^{\prime}(3) \mathcal{A}^{\prime}=\mathcal{A} \backslash\left\{\gamma, \mathcal{A}_{2}\right\} \cup$ $\left\{\#_{X_{1}: C_{12}}\left[\mathcal{A}_{12}\right]\right\}$, with $\mathcal{A}_{12}=\mathcal{A}_{1} \cup \mathcal{A}_{2}\left\{X_{2} \rightarrow X_{1}\right\}$, and $C_{12}=C_{1} \backslash\left(X_{1} \neq X_{2}\right)(4)$ for each valuation $(h(),. \mathbf{a})$ to $\left(\#_{X_{1}}\left[\mathcal{A}_{12}\right], \mathcal{A}^{\prime} \backslash \#_{X_{1}}\left[\mathcal{A}_{12}\right]\right)$ :

$$
\phi^{\prime}(h(.), \mathbf{a})=\prod_{\left(\mathbf{a}_{12}\right) \in \operatorname{range}\left(\mathcal{A}_{12}\right)} \phi\left(h_{\left[\mathcal{A}_{1}\right]}^{-\mathbf{a}_{1}}, \mathbf{a}_{2} ; \mathbf{a}\right)^{h\left(\mathbf{a}_{12}\right)}
$$

where $\mathbf{a}_{i}$ denotes the projection of the valuation $\mathbf{a}_{12}$ on $\mathcal{A}_{i}\left\{X_{2} \rightarrow X_{1}\right\}$, and the histogram $h^{-\mathbf{r}}$ is such that $h^{-\mathbf{r}}(\mathbf{r})=h(\mathbf{r})-1$, and $h^{-\mathbf{r}}\left(\mathbf{r}^{\prime}\right)=h\left(\mathbf{r}^{\prime}\right)$, for $\mathbf{r}^{\prime} \neq \mathbf{r}$.

Note that the second precondition can be established by merging CFs. Merging is thus an enabling operator for this conversion operator.

\section{Elimination Operations}

In the previous section, we presented the operators that introduce or merge CFs. In this section, we present a lifted sum-out operator that eliminates CFs, as well as an operator that aggregates the results after lifted sum-out.

\subsection{Sum-out by Counting}

We present an operation for summing out an atom inside counting formulas. This lifted operation sums out all the randvars represented by the atom from the model. It functions as a rewrite rule that removes the atom from a counting formula and has the sum-out operation of C-FOVE as a special case.

Example. Consider summing-out $A(X)$ from the parfactor $\phi\left(\#_{X}[A(X), B(X)]\right)$. By lifted sum-out we derive the parfactor $\phi^{\prime}\left(\#_{X}[B(X])\right.$, for which we define the potential function $\phi^{\prime}$ such that for each histogram $h^{\prime} \in \operatorname{range}\left(\#_{X}[B(X)]\right)$ :

$$
\phi^{\prime}\left(h^{\prime}\right)=\sum_{h \sim h^{\prime}} N u m\left(h \mid h^{\prime}\right) \phi(h)
$$

where $h \sim h^{\prime}$ denotes a histogram $h \in \operatorname{range}\left(\#_{X}[A(X), B(X)]\right)$ that is compatible with histogram $h^{\prime}$, that is, $h^{\prime}=h_{[B]}$. The quantity $N u m\left(h \mid h^{\prime}\right)$ equals the number of possible ways that a valuation to $R V(A(X))$ with the counts $h^{\prime}$, can be extended to a valuation to $R V(A(X), B(X))$ with the counts $h$.

The coefficient Num $\left(h \mid h^{\prime}\right)$ is defined based on the number Num $(h)$ of possible valuations to the randvars that result in a histogram $h$ : For each histogram $h=\left\{\left(r_{i}, n_{i}\right)\right\}_{i=1}^{r}$, with $\sum_{i} n_{i}=n$, we define $\operatorname{Num}(h)=\frac{n !}{\left(n_{1} !\right) \ldots\left(n_{r} !\right)}$, and $\operatorname{Num}\left(h \mid h^{\prime}\right)=\frac{\operatorname{Num}(h)}{\operatorname{Num}\left(h^{\prime}\right)}$. 
Note that sum-out removes an atom from the counting formula. Summing-out the last atom, such as $B(X)$ in the above example, results in an empty counting formula, for which we define the range as $\{($ Null, 0$)\}, \operatorname{Num}(($ Null, 0$))=1$, and which we trivially remove from the list of arguments after sum-out. The operation is formally defined below.

Operation: SUM-OUT

Input: (1) a parfactor $g=\forall \mathbf{L}: \phi(\mathcal{A}) \mid C$ in model $G(2)$ a counting formula $\gamma=\#_{X_{1}: C_{1}}\left[\mathcal{A}_{1}\right]$ in $\mathcal{A}(3)$ an atom $A \in \mathcal{A}_{1}$

Precondition: (1) logvar $(A)=\mathbf{L}(2)$ for all PRVs $\left(A^{\prime} \mid C^{\prime}\right)$ in the model, other than $\left(A \mid C \wedge C_{1}\right): R V\left(A \mid C \wedge C_{1}\right) \cap R V\left(A^{\prime} \mid C^{\prime}\right)=\emptyset$

Output: $g^{\prime}=\forall \mathbf{L}: \phi^{\prime}\left(\mathcal{A}^{\prime}\right) \mid C$, such that:

(1) $\mathcal{A}^{\prime}=\mathcal{A} \backslash\{\gamma\} \cup\left\{\gamma^{\prime}\right\}$, with $\gamma^{\prime}=\#_{X_{1}: C_{1}}\left[\mathcal{A}_{1} \backslash\{A\}\right]$ (2) for each valuation $\left(h^{\prime}(),. \mathbf{a}\right)$ to $\left(\gamma^{\prime}, \mathcal{A}^{\prime} \backslash \gamma^{\prime}\right)$ :

$$
\phi^{\prime}\left(h^{\prime}(.), \mathbf{a}\right)=\sum_{h \sim h^{\prime}} N u m\left(h \mid h^{\prime}\right) \cdot \phi(h(.), \mathbf{a})
$$

This operation has the sum-out operation of C-FOVE as a special case, namely when $\mathcal{A}_{1}=\{A\}$. It can also be further generalized to sum-out a group of atoms $\left\{A_{1}, \ldots, A_{n}\right\} \subseteq \mathcal{A}$, if the two preconditions are satisfied for all atoms.

\subsection{Aggregation}

In lifted inference, after dividing a problem into isomorphic subproblems, first the result of one prototypical instance is computed and then the result is aggregated, usually with the operation of exponentiation. This operator in fact multiplies a group of identical factors and is applied when a logvar disappears from the parfactor after a sum-out. Aggregation can, however, be extended to cases where a simple exponentiation does not work. In this section we extend this operator and show how this allows for more efficient lifted computations.

Example. Consider the parfactor $g$ of the form $\forall Y: \phi\left(\#_{X: X \neq Y}[P(X)], Q(Y)\right)$. Summing-out $Q(Y)$ results in the parfactor $g^{\prime}=\forall Y . \phi^{\prime}\left(\#_{X: X \neq Y}[P(X)]\right)$, on which sum-out is no longer applicable. Note that the logvar $Y$ is still part of the parfactor, although it does not appear in any atom. We show how, by aggregation, we can rewrite $g^{\prime}$ as an equivalent parfactor $g^{\prime \prime}=\phi^{\prime \prime}\left(\#_{X}[P(X)]\right)$, which is free of logvar $Y$. Assume $D(X)=D(Y)=\{a n n, b o b, c a r l$, dave $\}$. Then $g^{\prime}$ represents four ground factors, one for each person in $D(Y)$, e.g., for $Y=$ ann there is a factor $\phi^{\prime}\left(\#_{X: X \neq a n n}[P(X)]\right)$ in $g r\left(g^{\prime}\right) . g^{\prime \prime}$ should be defined such that $\phi^{\prime \prime}\left(\#_{X}[P(X)]\right)$ equals the product of these four factors. Note that these factors all have the same potential, but each on a CRV that excludes one distinct randvar from the group $R_{P}=\{P($ ann $), P(b o b), P($ carl $), P($ dave $)\}$. Given any assignment to $R_{P}$ with $n_{t}$ true and $n_{f}$ false, each of these CRVs has either one less true than $n_{t}$ or one less false than $n_{f}$. Specifically, there are $n_{t}$ histograms with counts $\left(n_{t}-1, n_{f}\right)$ and $n_{f}$ histograms with counts $\left(n_{t}, n_{f}-1\right)$. Since the value $\phi^{\prime}(h)$ is the same for all factors with the same histogram $h$, to compute the product it suffices to know $\left(n_{t}, n_{f}\right)$. Aggregation rewrites $g^{\prime}$ as $\phi^{\prime \prime}\left(\#_{X}[P(X)]\right)$, 
where the potential $\phi^{\prime \prime}$ is such that:

$$
\phi^{\prime \prime}\left(\left(n_{t}, n_{f}\right)\right)=\phi^{\prime}\left(\left(n_{t}-1, n_{f}\right)\right)^{n_{t}} \cdot \phi^{\prime}\left(\left(n_{t}, n_{f}-1\right)\right)^{n_{f}}
$$

The logvar $Y$ is now removed from $g^{\prime}$, and we can eliminate $P(X)$ from the model by lifted sum-out.

The more expensive alternative to aggregation is to apply counting on both atoms, and work with the parfactor $\phi^{*}\left(\#_{X}[P(X), Q(X)]\right)$. This alternative solution eliminates both $P$ and $Q$ atoms with counting sum-out, in poly time, while the above solution uses counting only for the $P$ atoms, and runs in linear time.

We formalize this operator as follows.

\section{Operation: AGGREGATE}

Input: (1) a parfactor $g=\forall \mathbf{L} \phi(\mathcal{A}) \mid C$ in model $G(2)$ a counting formula $\gamma=$ $\#_{X_{1}: C_{1}}\left[\mathcal{A}_{1}\right]$ in $\mathcal{A}(3)$ a logvar $X_{2} \in \mathbf{L} \backslash \operatorname{logvar}(\mathcal{A})$

Precondition: $\mathcal{A}$ has no counting formula $\#_{X_{i}: C_{i}}$ [.] other than $\gamma$, such that $\left(X_{i} \neq X_{2}\right) \in C_{i}$

Output: $g^{\prime}=\forall \mathbf{L}^{\prime}: \phi^{\prime}\left(\mathcal{A}^{\prime}\right) \mid C^{\prime}$, such that:

(1) $\mathbf{L}^{\prime}=\mathbf{L} \backslash\left\{X_{2}\right\}$ (2) $C^{\prime}$ is the projection of $C$ on $L^{\prime}$ (3) $\mathcal{A}^{\prime}=\mathcal{A} \backslash\{\gamma\} \cup$ $\left\{\#_{X_{1}: C_{1}^{\prime}}\left[\mathcal{A}_{1}\right]\right\}$, with $C_{1}^{\prime}=C_{1} \backslash\left\{X_{1} \neq X_{2}\right\}$ (4) for each valuation $(h(),. \mathbf{a})$ to $\left(\#_{X_{1}: C_{1}^{\prime}}\left[\mathcal{A}_{1}\right], \mathcal{A}^{\prime} \backslash\left\{\#_{X_{1}: C_{1}^{\prime}}\left[\mathcal{A}_{1}\right]\right\}\right)$ :

$$
\phi^{\prime}(h(.), \mathbf{a})=\prod_{\mathbf{a}_{1} \in \operatorname{range}\left(\mathcal{A}_{1}\right)} \phi\left(h^{-\mathbf{a}_{1}}(.), \mathbf{a}\right)^{h\left(\mathbf{a}_{1}\right)}
$$

\section{Relation to Joint Conversion}

Our contributions are closely related to, and target similar problems as, joint conversion and just-different counting conversion [1]. Our approach, however, can provide more efficient solutions than those based on the mentioned methods.

Joint conversion enables counting the states of a group of tuples of randvars, without modifying Milch et al.'s definition of counting formulas [4]. For instance, to enable counting tuples of randvars $(A(x), B(x))$, joint conversion replaces each occurrence of atoms $A(X)$ and $B(X)$ in the model with a joint atom $J_{A B}(X)$, whose state is the Cartesian product of the two atoms. Counting conversion can then derive a counting formula like $\#_{X}\left[J_{A B}(X)\right]$, which corresponds to a formula $\#_{X}[A(X), B(X)]$ in our formulation. When combined with just-different counting [1], joint conversion may also enable counting on logvars that are constrained to be unequal, similar to our approach.

However, there are differences between the two methods. Joint conversion is a global operation on the model, which introduces more dependencies by coupling two randvars into a joint randvar. After this operation, inference deals solely with the joint atom, and never directly with its constituents. Our method, however, uses a more fine grained formulation, by which it not only can simulate joint conversion, but also provide more efficient solutions than those possible by joint conversion. This primarily happens when the operations can divide the problem 
into independent parts, by eliminating a subset of the atoms that joint conversion couples in a joint atom. This allows for more efficient computations by avoiding the dependencies induced by unnecessary joint conversions. We illustrate this advantage in the following example.

Example. Consider a market domain involving a group of competing vendors $v_{1}, v_{2}, \ldots, v_{n}$. Let $P$ stand for PriceHigh, and let $P_{i}\left(v_{j}\right)$ represent a binary randvar corresponding to the event that at the $i$ th time step (e.g., $i$ th month) vendor $v_{j}$ sets a high price for the product. We can model this domain for $m$ time steps using the following set of parfactors.

$$
\begin{aligned}
g_{1} & =\phi_{1}\left(P_{1}(X), P_{2}(Y)\right) \mid X \neq Y \\
g_{2} & =\phi_{2}\left(P_{2}(Y), P_{3}(X)\right) \mid X \neq Y \\
& \ldots \\
g_{m} & =\phi_{m}\left(P_{m}(X), P_{m+1}(Y)\right) \mid X \neq Y
\end{aligned}
$$

where $\mathcal{D}(X)=\mathcal{D}(Y)=\left\{v_{1}, \ldots, v_{n}\right\}$. The task is to compute the partition function in this model, that is, to sum-out all the random variables.

Our approach. To sum out all the randvars, i.e., to eliminate all the atoms, our approach proceeds as follows. It first performs counting conversion on $Y$ in $g_{1}$ to derive $g_{1}^{\prime}=\phi^{\prime}\left(P_{1}(X), \#_{Y: Y \neq X}\left[P_{2}(Y)\right]\right)$. Next, it eliminates $P_{1}(X)$ from the model by lifted sum-out and aggregation, resulting in $g_{1}^{*}=\phi_{1}^{*}\left(\#_{Y}\left[P_{2}(Y)\right]\right)$. To prepare the model for summing-out $P_{2}(Y)$, we perform the following operations:

1. COUnt-CONVERT on logvar $Y$ in $g_{2}$ to derive $g_{2}^{\prime}=\phi_{2}^{\prime}\left(\#_{Y: Y \neq X}\left[P_{2}(Y)\right], P_{3}(X)\right)$

2. MERGE-COUNT on logvar $X$ in $g_{2}^{\prime}$ to derive $g_{2}^{\prime \prime}=\phi_{2}^{\prime \prime}\left(\#_{Y}\left[P_{2}(Y), P_{3}(Y)\right]\right)$

3. multiply $g_{1}^{*}$ and $g_{2}^{\prime \prime}$ to derive $g_{12}=\phi_{12}\left(\#_{Y}\left[P_{2}(Y), P_{3}(Y)\right], \#_{Y}\left[P_{2}(Y)\right]\right)$

4. MERGE the counting formulas to derive $g_{12}^{\prime}=\phi_{12}^{\prime}\left(\#_{Y}\left[P_{2}(Y), P_{3}(Y)\right]\right)$

Now from this parfactor, we sum-out $P_{2}(Y)$ and get a parfactor $g_{2}^{*}$ of the form $\phi_{12}^{*}\left(\#_{Y}\left[P_{3}(Y)\right]\right)$. Inference continues by eliminating $P_{3}$ from parfactors $g_{2}^{*}$ and $g_{3}$, in a similar way as it eliminated $P_{2}$ from $g_{1}^{*}$ and $g_{2}$. We repeat this procedure for all the remaining atoms $P_{i}$, until we eliminate the last atom $P_{m+1}$, which concludes the inference. The complexity of the procedure is proportional to the size of the largest potential it handles. For elimination of each atom $P_{i}$, the size of the largest potential we handle is $O\left(n^{4}\right)$, proportional to the number of histograms in the range of a counting formula $\#_{X}\left[P_{i}(X), P_{i+1}(X)\right]$. As there are $m$ atoms $P_{i}$, the whole procedure is in time $O\left(m n^{4}\right)$.

Joint conversion. Any solution based on joint conversion and just different counting conversion is less efficient than the above method. Here we present one such typical solution. Joint conversion first replaces the atoms $P_{1}$ and $P_{2}$ with a joint atom $J_{12}$, which represents the joint state of the atoms. This changes $g_{1}$ and $g_{2}$ respectively into $\phi_{1}^{\prime}\left(J_{12}(X), J_{12}(Y)\right) \mid X \neq Y$ and $\phi_{2}^{\prime}\left(J_{12}(Y), P_{3}(X)\right) \mid X \neq Y$. Still $J_{12}$ cannot be summed-out from the model, due to the free logvar $X$ in $g_{2}$. Just-different conversion, to derive a $\phi_{1}^{\prime \prime}\left(\#_{X}\left[J_{12}(X)\right]\right)$ is not helpful either. The only option is to continue applying joint conversions between atoms such as $J_{12 \ldots k}$ and $P_{k+1}$, to finally have only one joint atom $J_{1 \ldots m+1}$ in the model. 
Note that range $\left(J_{1 \ldots m+1}\right)=\{\text { true }, \text { false }\}^{m+1}$. The model would then consist of parfactors of the form $\phi_{i}\left(J_{1 \ldots m+1}(X), J_{1 \ldots m+1}(Y)\right) \mid X \neq Y$. By multiplying these $m$ parfactors into one, and then just-different conversion, we derive a parfactor $g^{*}=\phi^{*}\left(\#_{X}\left[J_{1 \ldots m+1}(X)\right]\right)$. Finally we can sum-out the counting formula $\gamma^{*}=\#_{X}\left[J_{1 \ldots m+1}\right]$ from $g^{*}$. The complexity of these operations is dominated by the manipulation of the counting formula $\gamma^{*}$ and is proportional to $\left|\operatorname{range}\left(\gamma^{*}\right)\right|$ which is $O\left(n^{2^{m}}\right)$. Comparing this complexity to the complexity of our approach, $O\left(m n^{4}\right)$, we see that our method can be much more efficient than Apsel and Brafman (2011)'s approach [1]. For example, for $m=10$, the latter approach has complexity $O\left(n^{1024}\right)$, in contrast to our approach which has complexity $O\left(10 n^{4}\right)$.

\section{Conclusion}

Counting is one of the fundamental techniques used in lifted inference to exploit the symmetry among interchangeable randvars. In LVE, this technique works by manipulation of counting formulas. We showed how generalizing the structure of counting formulas, along with the operators that manipulate them, provides more opportunities for lifting. Our approach is closely related to the method of joint conversion [1], but can offer more efficient solutions than this method.

Further generalizations of counting formulas, to capture more symmetries, are also conceivable. For instance, one can allow multiple counted logvars in a formula, instead of one. The semantics of such formulas is straightforward, e.g., $\#_{X Y}[P(X, Y), Q(X, Y)]$ aggregates the joint state of the tuples $(P(x, y), Q(x, y))$. However, manipulation of such formulas, especially counting the number of isomorphic states, can easily lead to non-trivial combinatorial problems, e.g., consider the formula $\#_{X Y Z}[P(X, Y), P(Y, Z)]$. Future research on such problems can bring valuable insights for lifted inference.

Acknowledgements This research is supported by the Research Fund K.U.Leuven (GOA 08/008,CREA/11/015 and OT/11/051), and FWO-Vlaanderen (G.0356.12).

\section{References}

1. Udi Apsel and Ronen I. Brafman. Extended lifted inference with joint formulas. In Proceedings of the 27th Conference on Uncertainty in Artificial Intelligence (UAI), pages 11-18. AUAI Press, 2011.

2. Rodrigo de Salvo Braz. Lifted first-order probabilistic inference. PhD thesis, Department of Computer Science, University of Illinois at Urbana-Champaign, 2007.

3. Lise Getoor and Ben Taskar, editors. An Introduction to Statistical Relational Learning. MIT Press, 2007.

4. Brian Milch, Luke S. Zettlemoyer, Kristian Kersting, Michael Haimes, and Leslie Pack Kaelbling. Lifted probabilistic inference with counting formulas. In Proceedings of the 23rd AAAI Conference on Artificial Intelligence (AAAI), pages 1062-1608, 2008.

5. David Poole. First-order probabilistic inference. In Proceedings of the 18th International Joint Conference on Artificial Intelligence (IJCAI), pages 985-991, 2003.

6. David Poole and Nevin Lianwen Zhang. Exploiting contextual independence in probabilistic inference. J. Artif. Intell. Res. (JAIR), 18:263-313, 2003. 